\title{
Accumulation of Hyaluronan and Tissue Edema in Experimental Myocardial Infarction
}

Anders Waldenström, * Hans Jörgen Martinussen, ${ }^{*}$ Bengt Gerdin, and Roger Hallgren*

Departments of ${ }^{*}$ Internal Medicine, ${ }^{\ddagger}$ Anesthesiology, and ${ }^{\$}$ Surgery, The University Hospital, S-751 85 Uppsala, Sweden

\begin{abstract}
Experimental myocardial infarction was induced in rats. The myocardial accumulation of hyaluronan (HA) and water during the development of infarction was measured. The extractable HA content of the infarcted area increased progressively from day 1 and on day 3 reached a threefold increase compared with the HA amounts in myocardium of sham operated controls. The relative water content of infarcted areas also increased progressively reaching a maximum value by day 3 and was strongly correlated with the HA accumulation. Affinity histochemistry visualized a thin rim of $\mathrm{HA}$ in the endoperimysium in healthy myocardium. By day 2 an interstitial edema with inflammatory cells was apparent. The widened endoperimysium stained extensively for HA. By its water-binding ability, interstitial accumulation of $\mathrm{HA}$ will contribute to the interstitial edema in infarcted myocardial tissue. An interstitial edema is likely to influence the electromechanical characteristics of the myocardium and facilitate reentry phenomena due to a loss of contact between muscle cells. The edema also induces an increased extracellular pressure and an altered myocardial wall compliance that might impair myocardial microcirculation. The findings are relevant to an understanding of the beneficial effect of hyaluronidase treatment in limiting cellular damage during myocardial ischemia. (J. Clin. Invest. 1991. 88:16221628.) Key words: hyaluronic acid - myocardial infarction • edema
\end{abstract}

\section{Introduction}

In acute myocardial infarction the ischemic area is subject to an inflammatory response with the appearance of white blood cells and formation of edema. This environment is the preparatory phase for the subsequent repair, which is characterized by remodelling of the connective tissue and synthesis of collagen. The early stage of formation and remodelling of the extracellular matrix has been little studied but is of importance not only in the development of granulation tissue for the subsequent repair but also in the final function of the damaged myocardium.

Accumulation of the connective tissue component hyaluronan (hyaluronic acid by older nomenclature), is an early event in tissue repair. Hyaluronan (HA) ${ }^{1}$ is a linear carbohydrate polymer and is an important constituent of loose connective

Address correspondence to Dr. Anders Waldenström, Department of Internal Medicine, University Hospital, S-751 85 Uppsala, Sweden. 1991.

Received for publication 30 May 1990 and in revised form 20 May

J. Clin. Invest.

(C) The American Society for Clinical Investigation, Inc.

0021-9738/91/11/1622/07 \$2.00

Volume 88, November 1991, 1622-1628 tissue $(1,2)$. The properties of HA are of importance in water homeostasis by attracting water and resisting water flow $(2,3)$. Thus, excessive accumulation of HA in interstitial tissue is related to an increased water content and thereby contributes to the interstitial edema seen in inflammatory states $(4,5)$. It has been suspected that $\mathrm{HA}$ is accumulated in infarcted tissue concomitantly with water retention (6) but the time of onset and duration of structural changes in myocardial HA are unknown. It may be hypothesized that HA synthesis is increased in critically ischemic tissue and that the water-binding properties of HA are of great importance to the development of edema in the ischemic myocardium, influencing both the mechanical and electrophysiological functions of the heart. We here present a study of the temporal accumulation of water and HA and its localization in infarcting rat heart tissue.

\section{Methods}

Male Sprague-Dawley rats weighing 550-690 g were anesthetized by placing the rats in a plastic container with an inlet tube for anesthetic gas and an outlet tube for evacuation of scavenger gases. For induction of anesthesia, $4 \%$ enflurane (Efrane ${ }^{\oplus}$; Abbott Laboratories, North Chicago, IL), 3 liters $\mathrm{N}_{2} \mathrm{O} / \mathrm{min}$ and 0.5 liters $\mathrm{O}_{2} / \mathrm{min}$ were used. When anesthetized, a mask with inlet and outlet tubes was placed over the nose and mouth and anesthesia was maintained with the mentioned gases during spontaneous breathing.

At day 0 the heart was exposed through a left lateral thoracotomy. A 5- 0 suture was inserted around the left anterior descending (LAD) artery. Before tying the ligature, pure oxygen with $1 \%$ enflurane was offered. The thoracotomy was thereafter closed in three layers. The sham operated animals obtained no LAD ligature, but were otherwise treated as the LAD ligated rats.

The rats were killed at day $1(n=4), 2(n=4), 3(n=5)$, or $4(n=6)$ after induction of myocardial ischemia and at day $1(n=2), 3(n=5)$, and $4(n=5)$ after sham operation. The rats were anesthetized with pentobarbital $(150 \mathrm{mg} / \mathrm{kg}$ i.p.) whereafter the hearts were rapidly extirpated. The apical part was first cut and used for histopathologic examination (see below). Thereafter, one biopsy, $\sim 3 \times 3 \times 3 \mathrm{~mm}$, was taken from the infarcted area in the anterior wall and another was taken from a normal area of the posterior wall. Care was taken to avoid biopsy material from the border zone of the infarct. The biopsies were weighed and freeze dried.

Heart tissue preparation, extraction of tissue hyaluronan, and calculation of water content. The procedure has been previously described (7). In brief, material for histology was fixed in $1 \%$ cetylpyridinium chloride buffered $4 \%$ formalin, $\mathrm{pH}$ 7.3. Material for tissue analysis of $\mathrm{HA}$ and water content was weighed on filter paper at room temperature (wet wt) and after lyophilization at $-80^{\circ} \mathrm{C}$ for $4 \mathrm{~d}(\mathrm{dry} \mathrm{wt})$. The relative water content of heart tissue was calculated according to the formula:

$$
\frac{\text { w.w. }- \text { d.w. }}{\text { w.w. }} \times 100
$$

1. Abbreviations used in this paper: d.w., dry weight; HA, hyaluronan; HABP, HA-binding region of the cartilage proteoglycan; LAD, left anterior descending. 
where w.w. is wet wt and d.w. is dry wt. The HA was extracted from freeze-dried cardiac tissue with $0.5 \mathrm{M} \mathrm{NaCl}$ for $16 \mathrm{~h}$. The samples were then centrifuged for $15 \mathrm{~min}$ at $2,000 \mathrm{~g}$. The supernatants were recovered and the HA concentrations analyzed in duplicate with a radiometric assay (Pharmacia Diagnostics, Uppsala, Sweden), according to principles previously outlined (8). Known amounts of HA were used to construct a standard curve. The variability of the assay was $<10 \%$.

Localization of hyaluronan in heart tissue. An avidin-enzyme, biotin-protein system was used for the detection of HA in tissue. The method is based upon the very specific interaction between HA and the protein core of the cartilage proteoglycan (9). The technique contains three major steps: $(a)$ the specific binding of biotin-labelled protein to HA in the fixed tissue; $(b)$ the binding of an avidin-enzyme complex to the biotin; and $(c)$ visualization of bound enzyme with a substrate.

The HA-binding region of the cartilage proteoglycan (HABP) was prepared by affinity chromatography on HA-Sepharose as earlier described (10). The purified protein was then linked to biotin according to the principles outlined by Ripellino and associates (11). To protect the HA-binding site on the HABP, HA used during the biotinylation was then removed by affinity chromatography on HA-Sepharose. HABP-biotin was stored at $-20^{\circ} \mathrm{C}$ at a concentration of $150-160 \mu \mathrm{g}$ protein/ml until used.

Before staining for HA, the heart tissues were dehydrated in ethanol, cleared in xylene, embedded in paraffin and transverse sections, 5 $\mu \mathrm{m}$ thick, were taken for staining. The staining for HA was performed according to the principles given by Ripellino and associates (11) and as previously described by us (7).

After incubation with biotinylated HABP, staining was performed using ABC Vectastain Reagent (Vector Laboratories, Inc., Burlingame, CA) using 3-amino-9-ethylcarbazol as substrate. The specificity of the reaction was checked with Streptomyces hyaluronidase (7). For histopathologic investigations, serial sections were also stained with hematoxylin-eosin.

Statistics. For statistical evaluation Student's paired $t$ test and analysis of variance were used. $P$ values $<0.05$ were considered statistically significant. Values are presented as means \pm SD. $n=$ indicates the number of animals.

\section{Results}

Histopathological features and localization of hyaluronan in the heart tissue (Fig. 1). A total of seven different rat hearts was investigated histologically. Two rats were sham operated and killed after $24 \mathrm{~h}$. One rat was killed at day 1 , three rats at day 2 , and one rat at day 3 after induction of myocardial infarction.

After staining with hematoxylin-eosin a subepicardial infiltration of inflammatory cells was observed on sections from all, even the sham operated, hearts and was interpreted as an unspecific response to surgery. In the sham operated animals the myocardium was undamaged without infiltration of inflammatory cells, with homogenous staining of myocytes and with well preserved cross striations.

At day 1 after LAD-ligation muscle fibers of the anterior wall were swollen and exhibited a variable degree of eosinophilic staining. In minor areas signs of hypercontraction were seen in isolated myocytes. There was also a scattered infiltration of inflammatory cells and microvascular stasis in the infarcted area.

At day 2 the infarcted area was clearly visible and there was an increased distance between myocytes, indicative of an increased water content. At this time it was obvious that LAD-ligation caused a very patchy infarction, which subendocardially encircled the entire left ventricle but was most pronounced in the anterior wall and transmural in a minor area only. In addition there were small microinfarctions in the form of patchy areas in the lateral and posterior walls.

At day 3 there was a widespread total necrosis of the myocytes within the infarcted area with the appearance of a large number of foam cell like macrophages.

The hyaluronan distribution in the sham operated animals was identical to that previously stated (7). There was thus a positive staining for hyaluronan in the very narrow space between the individual myocytes, the endomysium, but also in the coarser connective tissue fasciculi of the perimysium around groups of muscle cells. In addition, there was a positive staining for hyaluronan in the wide epimysial connective tissue and around vessels.

At day 1 after LAD-ligation there was an increased patchiness in the hyaluronan staining but it was not possible to definitely relate the more intensively stained regions to regions where muscle necrosis was morphologically seen at that time.

At day 2 the relative area of each section staining for hyaluronan was considerably increased in the infarcted zone. The staining was entirely located in the widened endomysium, i.e., the area for interstitial inflammation and edema (Fig. 1, $A$ and $B$ ).

At day 3 there was an intense staining for hyaluronan in the necrotic myocardium (Fig. 1, $C$ and $D$; left part of the figures). The widened interstitial space between preserved myocytes also stained intensely for hyaluronan. The negative staining of a serial section which was stained after incubation with Streptomyces hyaluronidase shows that the staining technique is specific for hyaluronan (Fig. $1 E$ ).

The hyaluronan content of the heart tissue (Fig. 2). The concentration of HA in the hearts of sham operated rats (n $=12)$ was similar in the anterior wall $(209 \pm 37 \mu \mathrm{g} / \mathrm{g} \mathrm{d}$.w. $)$ and in the posterior wall $(203 \pm 29 \mu \mathrm{g} / \mathrm{g} \mathrm{d}$.w.) as determined by extracting the freeze-dried and homogenized heart tissue with $0.5 \mathrm{M}$ $\mathrm{NaCl}$. There was no difference in anterior or posterior wall HA content between days 1,3 , and 4 in sham operated animals (analysis of variance). In the infarcted hearts $(n=19)$, the HA content of noninfarcted areas reached a maximum on day 2 $(492 \pm 119 \mu \mathrm{g} / \mathrm{g}$ d.w. $)$ and thereafter decreased. The HA content of infarcted areas reached its maximum on day $3(678 \pm 192$ $\mu \mathrm{g} / \mathrm{g}$ d.w.) and decreased on day 4 . At day 3 the HA content of the infarcted area was in the order of $75 \%$ higher than in the noninfarcted areas $(P<0.05)$, and three times higher than the sham operated animals $(P<0.001)$. The increase of HA in noninfarcted myocardium of hearts subjected to LAD ligation was statistically significant compared with the HA content in myocardium of sham operated animals.

The water content of heart tissue (Figs. 3 and 4). The relative water content in hearts of the sham operated rats $(n=12)$ was on average $75.1 \pm 0.6 \%$. There was no difference in water content between days 1,3 , and 4 in sham operated animals (analysis of variance). In the operated hearts $(n=19)$ the relative water content increased significantly in the infarcted area from day 1 to a maximum level by day $3(79.9 \pm 0.7 \%)$; the values were significantly higher both when compared with sham operated hearts and with the noninfarcted areas of the same heart. The relative water content of noninfarcted areas increased to a maximum level on day 3 of $77.4 \pm 0.3 \%$. The tissue content of HA exhibited a high degree of correlation ( $r$ $=0.71$ ) to the relative water content of the tissue. 

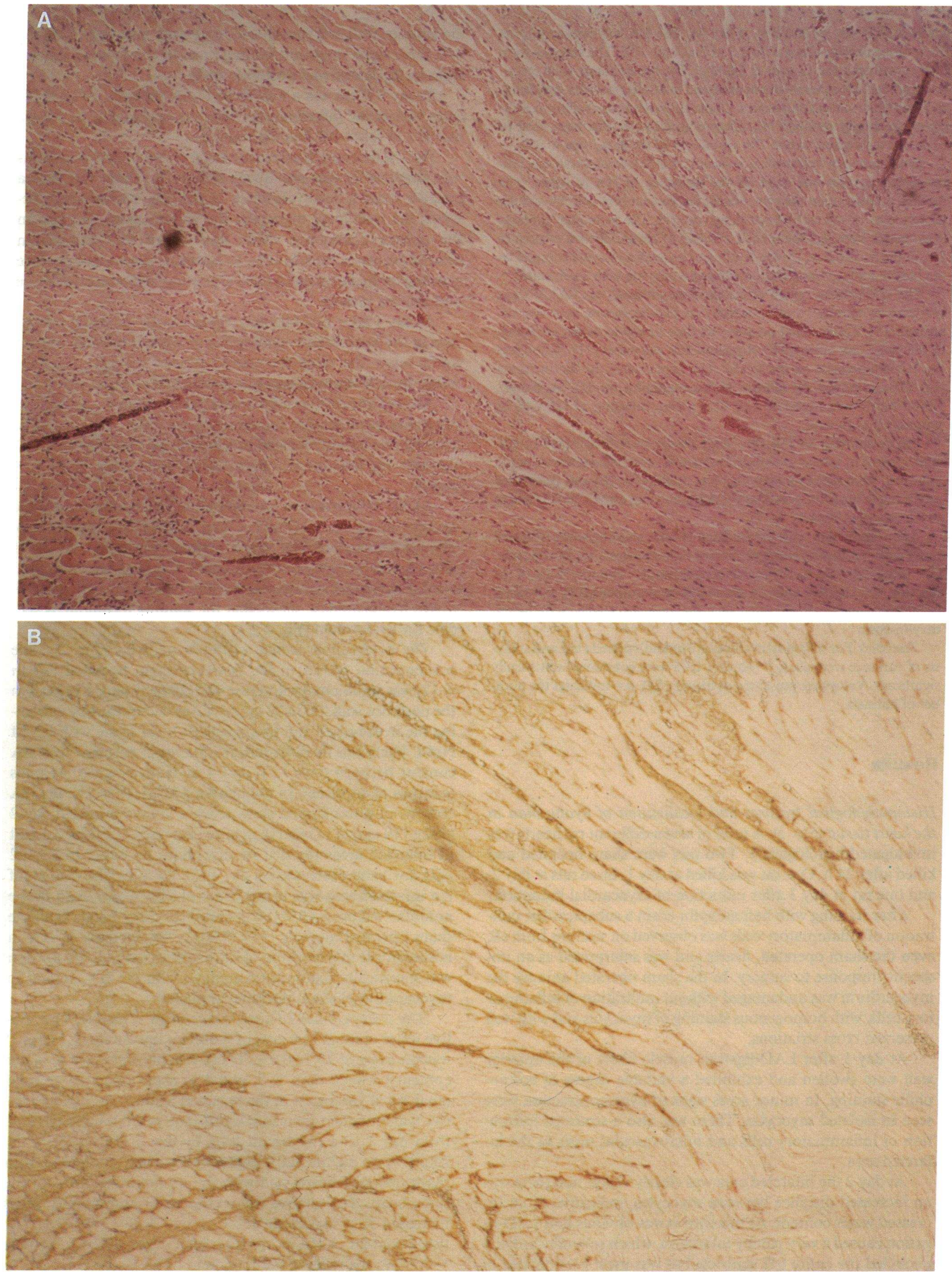

Figure 1 

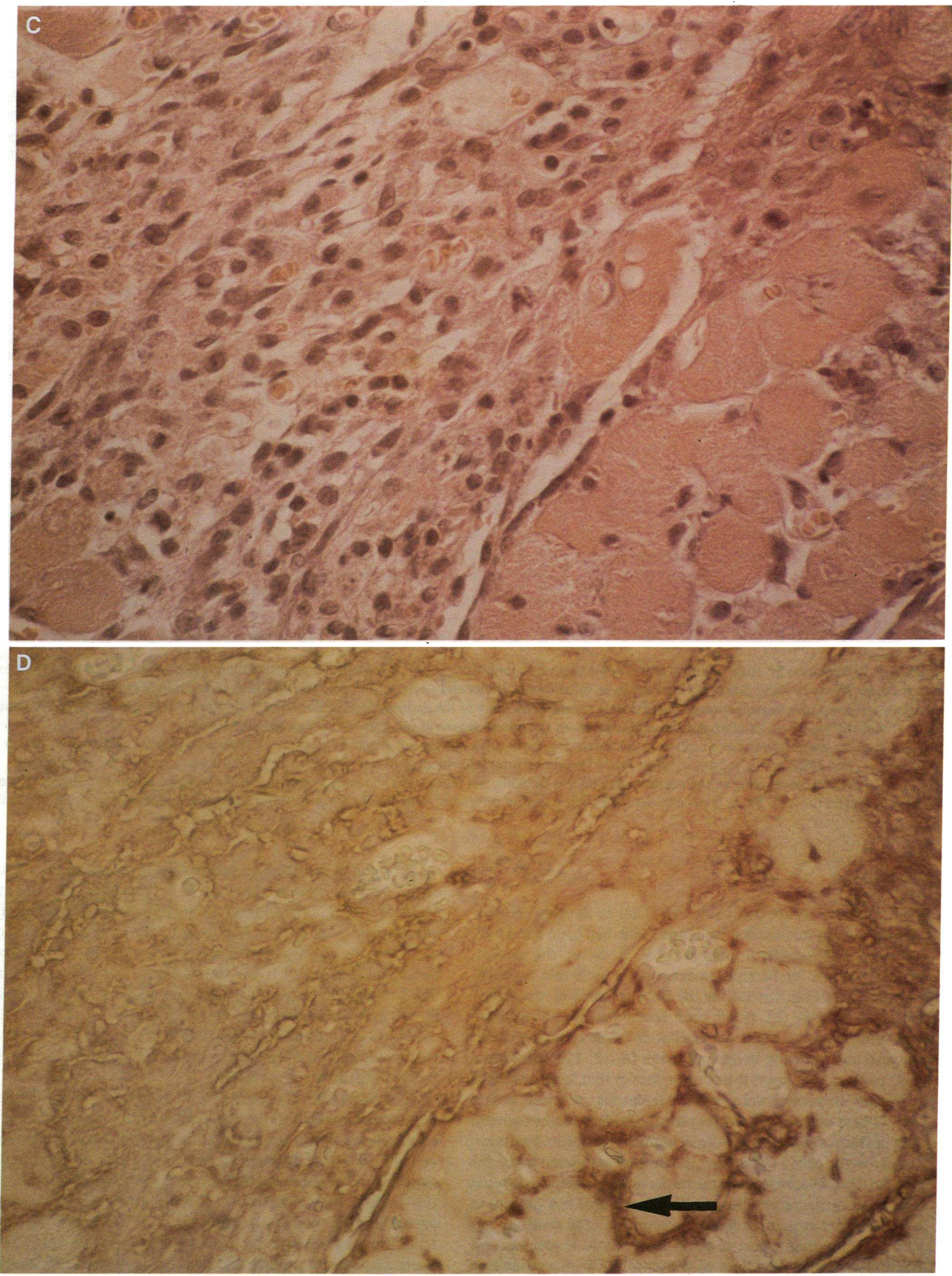

Figure 1 


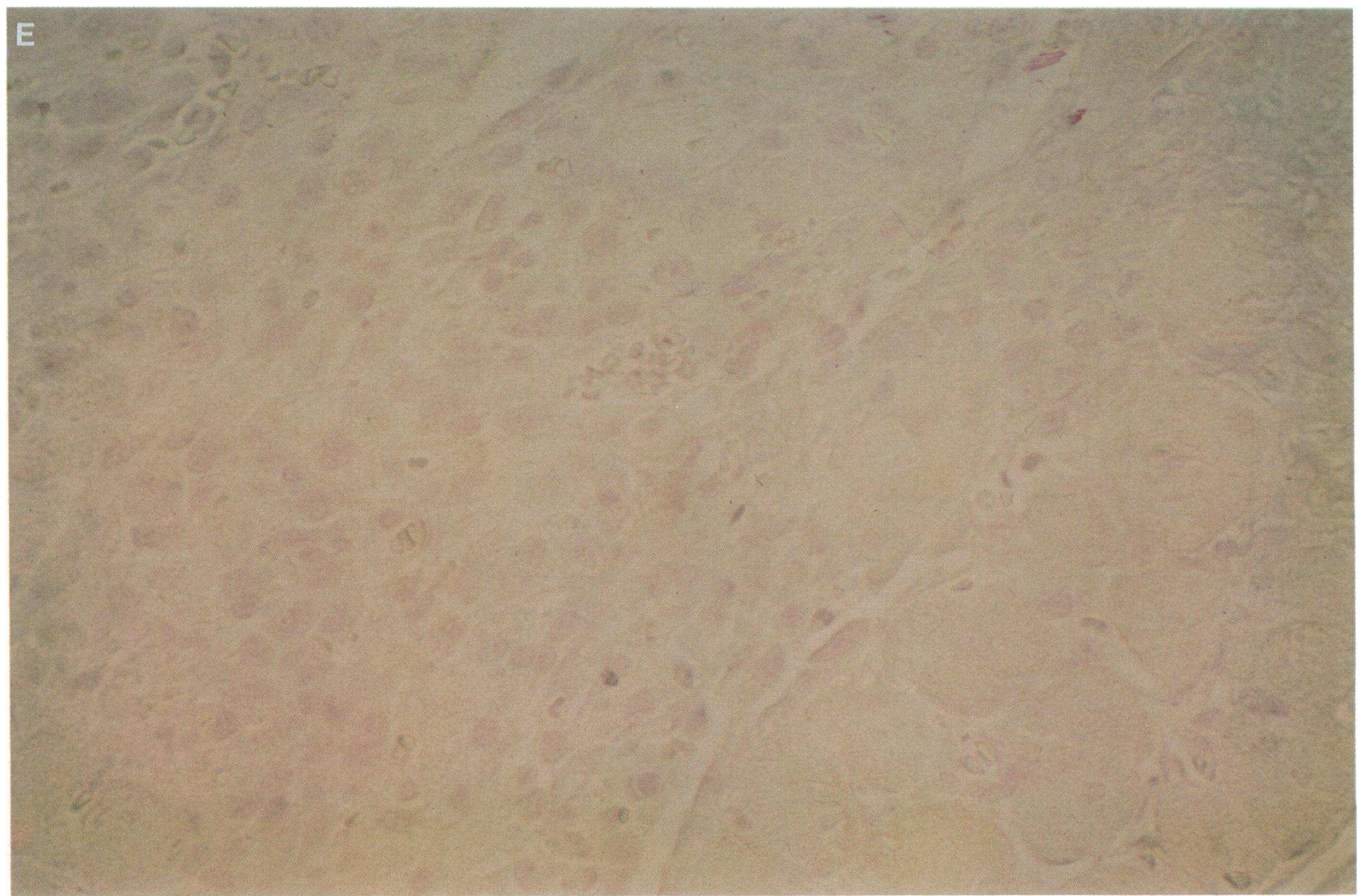

Figure 1. In hematoxylin-eosin staining of a section at postinfarction day $2(A)$ infarction area is seen in the upper left part, and normal myocardium at the lower right. Scattered infiltration of inflammatory cells and a widened endomysium is seen in the infarction area. In a serial section $(B)$ stained for visualization of HA the very restricted staining in the endomysium of the normal myocardium is seen in the lower right corner and the widespread staining of the endomysium of the infarcted area at top left. Original magnification $\times 64$.

In hematoxylin-eosin staining of a section at postinfarction day $3(C)$ a patchy total necrosis is seen with large amounts of inflammatory cell in the left part and preserved myocytes surrounded by certain endomysial widening in the right part. In a serial section stained for visualization of HA $(D)$ the intense staining of the necrotic area $(l e f t)$ is obvious, but there is also an intense staining of the widened epimysium between preserved myocytes (arrow). There is no staining for HA in a section pretreated with streptomyces hyaluronidase $(E)$. Original magnification $\times 400$.

\section{Discussion}

Hyaluronidase is reported to be beneficial in limiting cellular damage during myocardial ischemia. This has been shown in rats, dogs, and recently in man (12-15). However, the mechanisms by which hyaluronidase acts on the ischemic myocardium is still not known despite the fact that the first report was published in 1959 (6). It has been speculated that hyaluronidase reduces tissue water accumulation, thus reducing tissue pressure and allowing better perfusion $(6,12,15)$. In the isolated perfused rat heart it has been shown that hyaluronidase decreases interstitial volume of edematous ischemic hearts (16). Others have suggested that hyaluronidase might increase the diffusion of substrates to the ischemic cells and acid metabolites from the cells (13). So far, it seems to have been taken for granted that hyaluronidase exerts its action after myocardial infarction by degrading the normal amounts of HA present in the supportive structures of the myocardium. The results of our study add another aspect to the role of HA in myocardial infarction and shed new light to the action of hyaluronidase in this situation.
By using biotin-labelled proteoglycan core protein and an avidin-enzyme system (11), we have visualized HA in the healthy rat heart and followed the accumulation of HA in noninfarcted and infarcted rat heart tissue. The presence of most of the HA in the healthy heart in the epi- and perimysium reflects the established role of HA in the stabilization of the tissue matrix. In the infarcted area a progressive accumulation of HA in the edematous interstitium was apparent during the first four days after infarction. The microscopic findings of a progressive HA accumulation during infarction were confirmed by direct biochemical assay of tissue HA.

The increased concentration of $\mathrm{HA}$ in the noninfarcted myocardial tissue by day 2 after LAD ligation may have several explanations. First, the surgical trauma might affect HA kinetics for instance by altering lymph drainage from the myocardium. A second, probably more important, explanation is the finding that a thin subendocardial area was infarcted around the ventricular circumference. Third, there were small focal areas of necrosis seen in the septa, dorsal, and lateral walls, i.e., regions not affected by the LAD ligature. These microinfarctions, which were seen at day 1 after LAD ligation as 


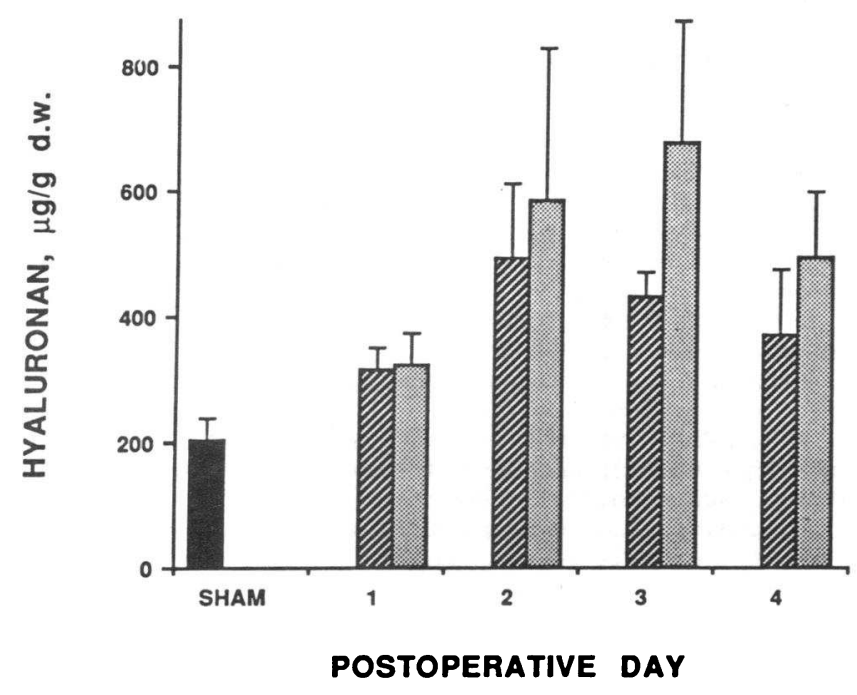

Figure 2. Myocardial concentration of hyaluronan in sham operated animals $(n=12)$ (average of anterior and posterior walls) and in noninfarcted and infarcted areas of hearts subjected to LAD ligation $(n=19)$. The rats were killed $1-4 \mathrm{~d}$ postoperatively. There was a significant difference between concentration of HA in sham operated hearts $(n=12)$ and concentration of HA in both noninfarcted and infarcted areas on days 2 and 3, respectively, and between noninfarcted and infarcted area on day 3. a, sham operated; $a$, noninfarcted; घ, infarcted.

areas of hypercontractions, may be secondary events due to stress-induced catecholamine release (17).

The cellular source of the myocardial HA production has not been identified in this study. It is conceivable that fibro-

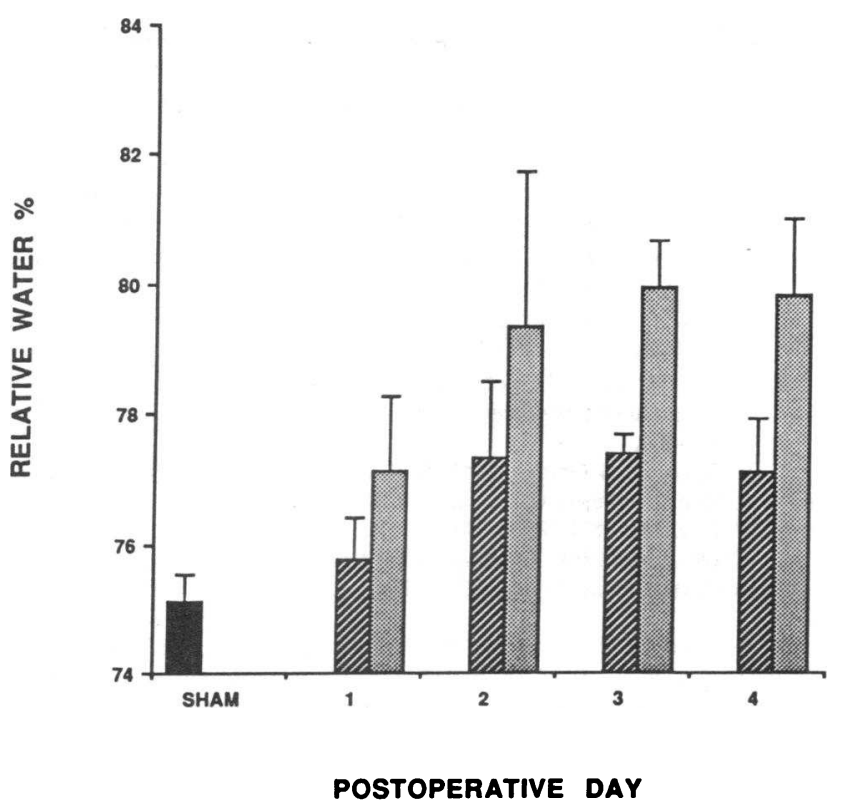

Figure 3. Myocardial water content in sham operated animals $(n$ $=12$ ) and in noninfarcted and infarcted areas of hearts subjected to LAD ligation $(n=19)$. The rats were killed $1-4 \mathrm{~d}$ postoperatively. There was a significant difference in water content between sham operated control hearts and water content in both noninfarcted and infarcted areas on day 3. The difference in water content between noninfarcted and infarcted areas was also significant on day 3. $m$, sham operated; $\mathrm{a}$, noninfarcted; $\mathrm{\theta}$, infarcted.

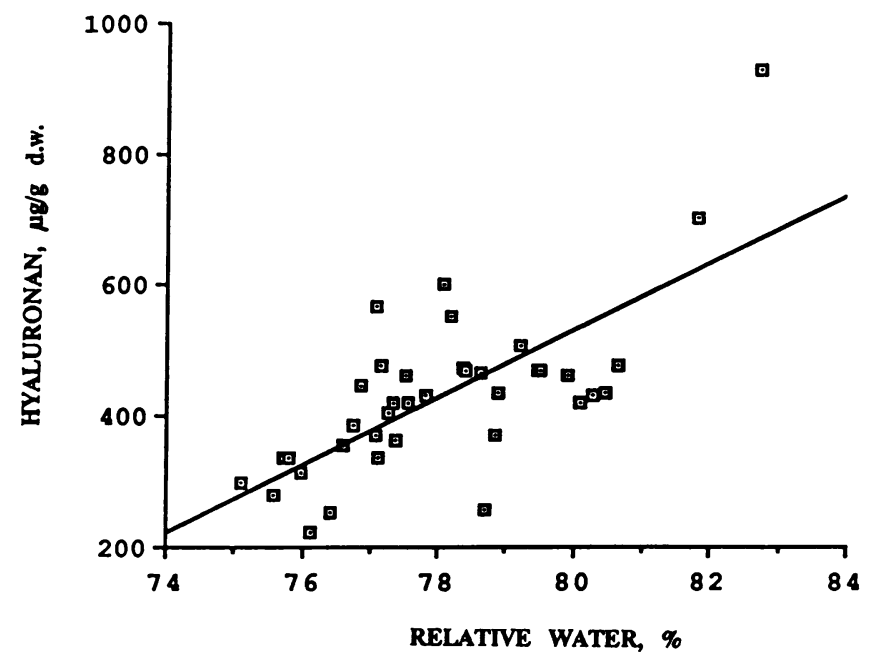

Figure 4. Correlation of water content vs. HA content $(r=0.71, P$ $<0.01$ ) in all myocardial biopsies taken from rats subjected to LAD ligation.

blasts or other mesenchymal cells present in the interstitial myocardial tissue become activated to an enhanced HA production by inflammatory mediators released by cells invading the infarct. Lymphokines and macrophage products can influence fibroblast activity $(18,19)$ and a number of inflammatory products with fibroblast-activating properties, e.g., interleukin 1 , platelet-derived and epidermal growth factors, have been shown to stimulate fibroblast synthesis of HA in vitro $(20,21)$. Hyaluronan has unique water-binding properties and is extremely hydrated. When the concentration of high molecular HA is higher than $0.2 \mathrm{mg} / \mathrm{ml}$, the molecules become entangled forming a network that occupies the solvent space and exclude large molecules (3). This phenomenon, called steric exclusion, may influence water transport and osmotic activity in the intercellular matrix (1). In our studies the concentration of $\mathrm{HA}$ at day 4 was $>600 \mu \mathrm{g} / \mathrm{g} \mathrm{d}$.w. in the infarcted tissue, which corresponds to $0.12 \mathrm{mg} / \mathrm{ml}$ wet vol. Assuming that the extra vascular space is in the order of $50 \%$ in the infarcted area this means that the concentration of $\mathrm{HA}$ in the myocardial interstitium will be $\sim 0.25 \mathrm{mg} / \mathrm{ml}$ and sufficient to permit steric exclusion to occur.

The increase in cardiac water after LAD ligature seems to be the combined result of the surgical trauma and the induced ischemia. Thus, by day 3 the water content had increased by $\sim 2 \%$ in the noninfarcted area and by $\sim 5 \%$ in the infarcted area. The increase in the water content was associated with an increase in the HA content. This finding offers indirect support for a link between HA and water accumulation in myocardial infarction. The accumulated HA is mainly localized in the interstitial space, which judged from the histology, is increased in volume. Tissue water of the infarcted areas, however, tends to increase before a rise of HA is seen. This is probably a combined effect of an intracellular edema due to failure of ion pumps, resulting in intracellular ion accumulation with ensuing osmotic swelling (22) and an increased microvascular permeability (23).

We have previously reported that a pronounced accumulation of HA in the smaller airways of patients with acute alveolitis is linked to indirect signs of alveolar interstitial edema (4). During experimental alveolitis an accumulation of HA in lung 
tissue also parallels development of interstitial edema (24). Furthermore, we have observed that the transplantation edema in rejecting hearts and kidneys is associated with an interstitial myocardial accumulation of $\mathrm{HA}(7,25)$. Thus, available data indicate that $\mathrm{HA}$ accumulation may occur as a general phenomenon in various organs attacked by inflammatory cells and seems to be implicated in the development of tissue edema.

The synthesis of a matrix rich in HA seems to be an important event during the early stages of the formation and remodelling of embryonic tissue and during wound repair (26). The matrix is thereby stabilized and cell infiltration and migration are stimulated (25-29). Thus, the early accumulation of HA and water in the myocardial interstitial tissue during infarction may facilitate the cell infiltration and scar formation. Development of edema in ischemic tissue might, however, be a threat to myocardial function due to compression of capillaries and to increased diffusion distance. On the other hand, Rovetto (12) did not find any change of glucose use in hyaluronidase-treated hearts although the lactate concentration was reduced. Hyaluronidase treatment obviously decreases HA content early in experimental infarction (13) and in parallel there is a decrease in water content (6). By inspecting the micrographs in our study it is easy to understand that HA accumulation and edema resulting in loss of contact between cells might be a good explanation for altered electromechanical characteristics and development of reentry phenomena.

In a recent trial, hyaluronidase was used as a treatment to myocardial infarction patients (15). Those patients who had early peaking of myocardium-specific creatine kinase in plasma suggestive of reperfusion showed reduced mortality. The authors suggested that the beneficial effect might be due to an altered repair or healing also discussed in this paper but again a normalized microcirculation may be an important part of this process. Therapeutic studies of hyaluronidase in experimental myocardial infarction are now underway with special reference to the inflammatory and connective tissue response and their influence on microcirculation.

\section{Acknowledgments}

Dr. Anders Tengblad (deceased) for the gift of biotinylated HABP. Ms. Marie Malmgren for excellent staining.

Supported by the Swedish Lung Heart Foundation, the Swedish Medical Research Council, and the Swedish Cancer Foundation.

\section{References}

1. Laurent, T. C. 1970. Structure of hyaluronic acid. In Chemistry and Molecular Biology of the Intercellular Matrix. E. A. Balazs, editor. Academic Press, New York and London. 702-732.

2. Mason, R. M. 1981. Recent advances in the biochemistry of hyaluronic acid in cartilage. Prog. Clin. Biol. Res. 54:87-112.

3. Comper, W. D., and T. C. Laurent. 1978. Physiological function of connective tissue polysaccharides. Physiol. Rev. 58:255-315.

4. Bjermer, L., A. Engström-Laurent, R. Lundgren, L. Rosenhall, and R. Hallgren. 1987. Hyaluronic acid and procollagen III peptide in bronchoalveolar lavage fluid as indicators of lung disease activity in farmer's lung. Br. Med. J. 295:803-806.
5. Nettelbladt, O., J. Bergh, M. Schenholm, A. Tengblad, and R. Hällgren. 1989. Accumulation of hyaluronic acid in the alveolar interstitial tissue in bleomycin-induced alveolitis. Am. Rev. Respir. Dis. 139:759-762.

6. Martins de Oliveira, J., R. Carballo, and H. A. Zimmerman. 1959. Intravenous injection of hyaluronidase in acute myocardial infarction: preliminary report of clinical and experimental observations. Am. Heart J. 57:712-722.

7. Hallgren, R., B. Gerdin, A. Tengblad, and G. Tufveson. 1990. Accumulation of hyaluronan (hyaluronic acid) in myocardial interstitial tissue parallels development of transplantation edema in heart allografts in rats. J. Clin. Invest. 85:668-673.

8. Tengblad, A. 1980. Quantitative analysis of hyaluronate in nanogram amounts. Biochem. J. 185:101-105.

9. Hascell, V. C. 1977. Interaction of cartilage proteoglycans with hyaluronic acid. J. Supramol. Struct. 7:101-120.

10. Tengblad, A. 1979. Affinity chromatography on immobilized hyaluronate and its application to the isolation of hyaluronate binding proteins from cartilage. Biochim. Biophys. Acta. 578:281-289.

11. Ripellino, J. A., M. M. Klinger, R. U. Margolis, and R. K. Margolis. 1985. The hyaluronic acid binding region as a specific probe for the localization of hyaluronic acid in tissue sections. J. Histochem. Cytochem. 33:1060-1066.

12. Rovetto, M. J. 1977. Effect of hyaluronidase and methylprednisolone on myocardial function, glucose metabolism and coronary flow in the isolated ischemic rat heart. Circ. Res. 41:373-379.

13. Maroko, P. R., D. M. Davidson, P. Libby, A. D. Hagan, and E. Braunwald. 1972. Reduction by hyaluronidase of myocardial necrosis following coronary artery occlusion. Circulation. 46:430-437.

14. Maclean, D., M. C. Fishbein, P. R. Maroko, and E. Braunwald. 1976 Hyaluronidase-induced reduction in myocardial infarct size. Science (Wash. DC). 194:199-200.

15. Roberts, R., E. Braunwald, J. E. Muller, C. Crof, H. K. Gold, T. D. Hartwell, A. S. Jaffe, S. M. Mullin, C. Parker, E. R. Passamani, et al. 1988. Effect of hyaluronidase on mortality and morbidity in patients with early peaking of plasma creatine kinase MB and non-transmural ischaemia. Br. Heart J. 60:290298.

16. Sunnergren, K. P., and M. J. Rovetto. 1985. The effects of hyaluronidase on interstitial hydration, plasma protein exclusion, and microvascular permeability in the isolated perfused rat heart. Microvasc. Res. 30:286-297.

17. Haft, J., and K. Fani. 1973. Stress and the induction of intravascular platelet aggregation in the heart. Circulation. 48:164-169.

18. Johnson, R. L., and M. Ziff. 1976. Lymphokine stimulation of collagen accumulation. J. Clin. Invest. 58:240-252.

19. Wahl, S. M., and J. B. McCarthy. 1978. Lymphocyte-mediated activation of fibroblast proliferation and collagen production. J. Immunol. 121:942-946.

20. Hamerman, D., and D. D. Wood. 1984. Interleukin 1 enhances synovial cell hyaluronate synthesis. Proc. Soc. Exp. Biol. Med. 177:205-210.

21. Engström-Laurent, A., N. Feltelius, R. Hällgren, and P. Wasteson. 1985. Elevated serum hyaluronate in scleroderma. An effect of growth factor induced activation of connective tissue cells? Ann. Rheum. Dis. 44:614-620.

22. Michell, R. H., and R. Coleman. 1979. Structure and permeability of normal and damaged membranes. In Enzymes in Cardiology. Diagnosis and Research. D. J. Hearse and J. De Leiris, editors. John Wiley \& Sons, Chichester, New York, Brisbane, Toronto. 59-79.

23. Tucker, V. L., and V. H. Huxley. 1988. $\mathrm{O}_{2}$ modulation of single vessel hydraulic conductance. Am. J. Physiol. 254:H317-H323.

24. Nettelbladt, O., A. Tengblad, and R. Hällgren. 1989. Lung accumulation of hyaluronan parallels pulmonary edema in experimental alveolitis. Am. J. Physiol. 257:L379-384.

25. Hällgren, R., B. Gerdin, and G. Tufveson. Hyaluronic acid accumulation and redistribution in rejecting rat kidney graft: relationship to the transplantation edema. J. Exp. Med. 171:2063-2076.

26. Toole, B. P. 1981. Glycosaminoglycans in morphogenesis. In Cellular Biology of Extracellular Matrix. E. D. Hay, editor. Plenum Publ. Corp., NY. 251-294.

27. Le Dourain, N. M. 1984. Cell migrations in embryos. Cell. 38:353-360.

28. Tucker, R. P., and C. A. Erickson. 1984. Morphology and behaviour of quail neural crest cells in artificial three-dimensional extra-cellular matrices. Dev. Biol. 104:390-405.

29. Stern, C. D. 1984. Mini-review; hyaluronidases in early embryonic development. Cell Biol. Int. Rep. 8:703-717. 